\title{
Organizational dynamics in adaptive distributed search processes: effects on performance and the role of complexity\#
}

\author{
Friederike WALL \\ (Department of Controlling and Strategic Management, Alpen-Adria-Universitaet Klagenfurt, 9020 Klagenfurt, Austria) \\ E-mail: friederike.wall@aau.at \\ Received Sept. 21, 2015; Revision accepted Jan. 5, 2016; Crosschecked Mar. 9, 2016
}

\begin{abstract}
In this paper, the effects of altering the organizational setting of distributed adaptive search processes in the course of search are investigated. We put particular emphasis on the complexity of interactions between partial search problems assigned to search agents. Employing an agent-based simulation based on the framework of NK landscapes we analyze different temporal change modes of the organizational set-up. The organizational properties under change include, for example, the coordination mechanisms among search agents. Results suggest that inducing organizational dynamics has the potential to increase the effectiveness of distributed adaptive search processes with respect to various performance measures like the final performance achieved at the end of the search, the chance to find the optimal solution of the search problem, or the average performance per period achieved during the search process. However, results also indicate that the mode of temporal change in conjunction with the complexity of the search problem considerably affects the order of magnitude of these beneficial effects. In particular, results suggest that organizational dynamics induces a shift towards more exploration, i.e., discovery of new areas in the fitness landscape, and less exploitation, i.e., stepwise improvement.
\end{abstract}

Key words: Agent-based simulation, Complexity, Coordination, Distributed search, NK landscapes http://dx.doi.org/10.1631/FITEE.1500306

CLC number: TP13

\section{Introduction}

Adaptive distributed search processes occur in a large variety of real-world systems like, for example, networks of robots, 'swarms' of unmanned aerial vehicles (UAVs), or managers of firms collaboratively searching for higher levels of business performance. The question of how to organize these search processes, and, in particular, how to coordinate among distributed agents, is of fundamental relevance and

\footnotetext{
\# A preliminary version was presented at the 12 th International Conference on Distributed Computing and Artificial Intelligence, June 3-5, 2015, Spain

(B) ORCID: Friederike WALL, http://orcid.org/0000-0001-80018558

(C)Zhejiang University and Springer-Verlag Berlin Heidelberg 2016
}

has been investigated in various disciplines, e.g., complex systems science, robotics, and computational organization theory (Carley and Gasser, 1999; Gross and Blasius, 2008; Cao et al., 2013).

In this paper, we focus on the temporality of the organizational set-up for adaptive search processes. In particular, we are interested in the effects of inducing organizational dynamics, in terms of altering the organizational set-up, on the effectiveness of distributed adaptive search processes. This paper is an extended version of Wall (2015); in particular, the extensions comprise a detailed analysis of the role of complexity of search problems assigned to distributed agents and also reflect situations in which the level of complexity is unstable. With 
this, our research effort relates to the broad field of adaptive evolutionary networks; however, it is worth mentioning that the paper focuses mainly on 'dynamics of networks' rather than on 'dynamics on networks' (Gross and Blasius, 2008). With respect to distributed multi-agent coordination, research has been categorized into streams on consensus, formation control, optimization, task assignment, and estimation (Cao et al., 2013). The research effort presented here is directed towards the aspect of estimation, meaning that - since, for example, global information on the search problem is not available - a distributed search and assessment of partial solutions to the search problem are required. Hence, it is not the center of our research to figure out the performance of, for example, certain consensus mechanisms in dynamic task environments; rather, the effects of different modes of changing the organizational set-up in the course of adaptive search processes are the focus.

However, in various domains it is well known that the complexity of a search problem is of critical relevance for the performance achieved within distributed search processes - be it in the design of parallel search algorithms in general (Karp et al., 1988), the collaborative search in the context of certain optimization problems like, for example, the traveling salesman problem (Peterson, 1990), or the organizational design of firms (Thompson, 1967; Hansen, 1999). A key issue is whether it is possible to segment the overall search problem into disjoint partial problems and delegate these to different search agents. Whenever this is not possible or not appropriate, dependencies across the agents' partial search problems occur, which might cause performance losses if not considered adequately. Hence, a major issue in the organizational design of distributed search processes is how to deal with the complexity of cross-agent interactions.

Hence, we analyze the effects of organizational dynamics and put special emphasis on the role of cross-agent complexity of interactions. For this, we employ an agent-based simulation and, in particular, observe adaptive search processes conducted collaboratively by the agents of a search system whose organization is undergoing change. Our agents operate on fitness landscapes and seek to find superior levels of fitness or, in other terms, of the search system's performance. Our fitness landscapes are set up following the idea of NK fitness landscapes which were initially introduced in evolutionary biology (Kauffman and Levin, 1987; Kauffman, 1993). One of the major advantages of the NK framework is that it allows rather 'pure' control for the complexity of search problems (Altenberg, 1997), and probably for this reason it has been widely used to analyze the role of complexity in various domains (Wall, 2016a). The distinctive feature of our model is that it employs different temporal modes of mid-term dynamics imposed on the organizational setting of the search processes for higher levels of performance where the underlying search problem could show different levels of complexity. In particular, in our model organizational structures undergo randomized, multiple, and multidimensional changes.

\section{Simulation model}

In our simulations, artificial organizationsconsisting of search agents and endowed with some arrangements for coordination among agentssearch for superior levels of performance in the course of adaptive walks on NK fitness landscapes (Kauffman and Levin, 1987; Kauffman, 1993). To this end, our search systems use two means - in the short-term they stepwise search for better solutions to a given search problem for improving performance, and in the mid-term they can change their organizational set-up with respect to multiple dimensions of organizational design.

\subsection{Search problem and complexity}

In line with the standard NK model, in each time step $t$ of the observation period, our search systems under investigation face an $N$-dimensional binary search problem, i.e., they have to find a configuration of $d_{i t} \in\{0,1\}$, where $i=1,2, \ldots, N$ (for an extension of the standard binary case to mixed integer NK landscapes see Li et al. (2006)). Hence, at each time step, the search space consists of $2^{N}$ different binary vectors $\boldsymbol{d}_{t} \equiv\left(d_{1 t}, d_{2 t}, \ldots, d_{N t}\right)$. $d_{i t}$ might, for example, represent a single choice of an $N$-dimensional decision problem. Each of the two states $d_{i t} \in\{0,1\}$ makes a certain contribution $C_{i t}$ to the overall fitness $V\left(\boldsymbol{d}_{t}\right)$ of the organization. $C_{i t}$ is randomly drawn from a uniform distribution with $0 \leq C_{i t} \leq 1$.

The NK framework allows for representing interactions among choices with level $K . K$ reflects 
the number of those other choices $d_{j t}(j \neq i)$ which also affect the fitness contribution $C_{i t}$ of choice $d_{i t}$. $K$ can take values from 0 (no interactions) to $N-1$ (maximum interactions) and, for the sake of simplicity and in line with the original NK framework, we assume that $K$ is the same for all $d_{i}$ 's. Hence, the fitness contribution $C_{i t}$ might not only depend on the single choice $d_{i t}$ but also on $K$ other choices $d_{j t}$ where $j \in\{1,2, \ldots, N\}$ and $j \neq i$ :

$$
C_{i t}=f_{i}\left(d_{i t}, d_{j t}\right), j \in\{1,2, \ldots, N\}, j \neq i .
$$

The overall fitness (performance) $V_{t}$ achieved in period $t$ results as the normalized sum of contributions $C_{i t}$ from

$$
V_{t}=V\left(\boldsymbol{d}_{t}\right)=\frac{1}{N} \sum_{i=1}^{N} C_{i t}
$$

\subsection{Organizational set-up}

1. Search agents and central agent

The search processes are conducted in a distributed way. In particular, the $N$-dimensional search problem is segmented into $M$ disjoint partial problems where each of these subproblems is delegated to one search agent superscripted by $r$ $(r=1,2, \ldots, M)$ correspondingly. Each search agent has primary control of its 'own' subset of the $N$ choices. Hence, from the perspective of search agent $r$ the search problem is partitioned into a partial search vector $\boldsymbol{d}_{t}^{r}$ for those choices which are in its 'own' responsibility and into $\boldsymbol{d}_{t}^{r, \text { res }}$ for the residual choices that the other search agents $q \neq r$ are in charge of.

However, in case of interactions which go beyond the 'own' subproblem, choices of search agent $r$ might affect the contributions of the other agents' choices and vice versa. To capture the level of cross-agent complexity of interactions - which relates to the very core of our research question (Section 1) - we introduce $K^{*}$ which gives the number of single choices $d_{i}$ which, from the perspective of search agent $r$, are in the primary control of other search agents $q \neq r$ (with $0 \leq K^{*} \leq K-1$ ). For the sake of simplicity, we assume that the level of cross-agent interactions is the same for all search agents (hence, $K^{*}$ is not superscripted by $r$ ).

In addition to the decentralized search agents our search systems have a kind of 'central agent' whose particular role within the search process depends on the mode of coordination which we describe in more detail below.

2. Shaping the search agents' perspective

In each time step $t$, a search agent $r$ seeks to identify the best configuration for the 'own' subset of $N^{r}$ choices $\boldsymbol{d}_{t}^{r}$ assuming that the other agents do not alter their prior choices. In particular, an agent randomly discovers two alternatives of configurations for the subproblem assigned to this agent: an alternative configuration that differs in one choice $(a 1)$ and another alternative $(a 2)$ where two bits are flipped compared to the current configuration. Hence, together with the status quo $\boldsymbol{d}_{t-1}^{r *}$ and the two alternatives $\boldsymbol{d}_{t}^{r, a 1}$ and $\boldsymbol{d}_{t}^{r, a 2}$, agent $r$ has three options to choose.

However, which option a search agent favors, depends on the 'perspective' $P_{t}^{r}$ the agent has on the search problem or, in other words, how the agent's objective for the evaluation of options is shaped. An agent might focus only on the 'own' partial problem or may also take the rest of the search problem into consideration. This is controlled by parameter $\alpha^{r}$ in Eq. (3):

$$
P_{t}^{r}\left(\boldsymbol{d}_{t}\right)=P_{t}^{r, \text { own }}\left(\boldsymbol{d}_{t}^{r}\right)+\alpha^{r} \cdot P_{t}^{r, \text { res }},
$$

where

$$
P_{t}^{r, \text { own }}\left(\boldsymbol{d}_{t}^{r}\right)=\frac{1}{N} \sum_{i=1+p}^{N^{r}} C_{i t}
$$

with $p=\sum_{s=1}^{r-1} N^{s}$ for $r>1$ and $p=0$ for $r=1$, and

$$
P_{t}^{r, \text { res }}=\sum_{q=1, q \neq r}^{M} P_{t}^{q, \text { own }} .
$$

3. Coordination among search agents

The search system can employ three modes of coordination among the search agents:

(1) In a fairly decentralized mode each search agent decides on its 'own' partial choices $\boldsymbol{d}_{t}^{r}$ autonomously, and the overall configuration $\boldsymbol{d}_{t}$ results in a combination of these choices without any intervention by the central agent.

(2) As a type of horizontal coordination our search agents inform each other about their preferences from the three options $\boldsymbol{d}_{t-1}^{r *}, \boldsymbol{d}_{t}^{r, a 1}$, and $\boldsymbol{d}_{t}^{r, a 2}$; then the search agents combine their preferred partial configurations and each search agent evaluates the resulting composed configuration $\boldsymbol{d}_{t}$ according to 
its 'own' perspective on the search problem as shaped by parameter $\alpha^{r}$ in Eq. (3). The search agents have mutual veto power. Hence, a search agent vetoes an alteration of $\boldsymbol{d}_{t}$ which it expects to result in a lower $P_{t}^{r}\left(\boldsymbol{d}_{t}\right)$ compared to the status quo. In other words, a new configuration is selected only if it promises a higher $P_{t}^{r}\left(\boldsymbol{d}_{t}\right)$ to, at least, one agent than the status quo, and no agent is worse off with the new configuration compared with the status quo. The central agent does not intervene in decision making.

(3) In a rather centralized mode of coordination each search agent $r$ transfers a list with the two most preferred options from $\boldsymbol{d}_{t-1}^{r *}, \boldsymbol{d}_{t}^{r, a 1}$, and $\boldsymbol{d}_{t}^{r, a 2}$ to the central agent. The central agent then chooses that combination of the $M$ lists of proposals which promises the highest overall fitness $V$.

4. Increasing diversity of search

There is some evidence that imperfect information on the outcome (fitness, performance) of options could have beneficial effects on search processes (Levitan and Kauffman, 1995; Wall, 2010; 2013). In particular, the more rugged a fitness landscape, as shaped by the level of interactions given by $K$, the more likely it becomes that adaptive search processes employing local search stick to local maxima; falsepositve evaluations of options provide the opportunity to leave a local peak and, by that, to eventually find higher levels of fitness. In order to make use of these findings, we endow our agents, eventually, with slightly distorted information about the fitness of options. In particular, $P_{t}^{r \text {,own }}\left(\boldsymbol{d}_{t}^{r}\right)$ and $P_{t}^{r \text {,res }}\left(\boldsymbol{d}_{t}^{r}\right)$ (search agents), and $V_{t}$ (central agent) are distorted by adding error terms respectively:

$$
\begin{gathered}
\tilde{P}_{t}^{r, \text { own }}\left(\boldsymbol{d}_{t}^{r}\right)=P_{t}^{r, \text { own }}\left(\boldsymbol{d}_{t}^{r}\right)+e^{r, \text { own }}\left(\boldsymbol{d}_{t}^{r}\right), \\
\tilde{P}_{t}^{r, \text { res }}\left(\boldsymbol{d}_{t}\right)=P_{t}^{r, \text { res }}\left(\boldsymbol{d}_{t}\right)+e^{r \text {,res }}\left(\boldsymbol{d}_{t}\right),
\end{gathered}
$$

resulting in

$$
\tilde{P}_{t}^{r}\left(\boldsymbol{d}_{t}\right)=\tilde{P}_{t}^{r, \text { own }}\left(\boldsymbol{d}_{t}^{r}\right)+\alpha^{r} \cdot \tilde{P}_{t}^{r, \text { res }}\left(\boldsymbol{d}_{t}\right),
$$

and

$$
\tilde{V}_{t}\left(\boldsymbol{d}_{t}\right)=V_{t}\left(\boldsymbol{d}_{t}\right)+e^{\mathrm{cent}}\left(\boldsymbol{d}_{t}\right) .
$$

We reflect distortions as relative errors imputed to the true performance (for other functions see Levitan and Kauffman (1995)), and, for simplicity, the error terms follow a Gaussian distribution $N(0, \sigma)$ with expected value 0 and standard deviation $\sigma$. In particular, standard deviations $\sigma^{r, \text { own }}$ and $\sigma^{r \text {,res }}$, for the sake of simplicity, are assumed to be the same for search agents $r$ and stable in time. The latter also holds for $\sigma^{\text {cent }}$ which is relevant for the central agent. Errors are assumed to be independent of each other.

\subsection{Organizational dynamics}

In the center of our research effort is the question whether altering the organizational set-up of the search processes could increase the fitness of solutions achieved. The model captures three temporal modes of change:

1. 'Once': The organizational set-up is modified once in period $T^{*}$.

2. 'Periodical': The set-up is altered periodically after $T^{* *}$ periods.

3. 'Fitness-driven': The set-up is changed depending on the fitness increase in the previous periods, i.e., in every $T^{* * *}$ th time step the fitness change $\Delta V=V_{t}-V_{t-T^{* * *}}$ is assessed and, if $\Delta V$ is below a certain threshold $v$, then the set-up is altered.

Alterations of the organizational set-up can be put forward along three dimensions:

1. The objective (or perspective) of the distributed search agents as given in Eq. (3) can be modified in a range between focusing overall fitness $V_{t}$ and the fitness achieved with respect to the partial search problem $\boldsymbol{d}_{t}^{r}$ only (To be more precise, then parameter $\alpha^{r}$ in Eqs. (3) and (8), respectively, would have to be subscripted by $t$ ).

2. The mode of coordination can be changed between the three modes as introduced above.

3. The precision of ex ante-evaluation as given by $\sigma^{r \text {,own }}, \sigma^{r \text {,res }}$, and $\sigma^{\text {cent }}$ for the information errors can be modified (With this, the error terms in Eqs. (6), (7) and (9) could be subscripted by $t$ to be more precise).

The simulation model allows alterations in two randomly selected dimensions out of these three dimensions in all of the temporal change modes under investigation. Moreover, within each of these dimensions the alternative set-up is chosen randomly. This also applies to the initial organizational set-up of the distributed search processes.

\section{Simulations and parameter settings}

To simulate the search processes under the regime of different temporal modes of change, after 
a fitness landscape is generated, the initial organizational set-up of the search system is determined randomly. Then the systems are placed randomly in the fitness landscape and observed while searching for higher levels of overall fitness and-from time to time - changing their organizational set-up.

However, the very core of our research effort is to gain a closer understanding of the role of the search problem's complexity in the effectiveness of changing the organizational set-up of the search process. In order to reflect this research focus appropriately, our simulations are put forward in three steps:

First, we compare the effects of organizational dynamics for two rather 'extreme' types of search problems against each other which, in the following, are named the 'baseline scenarios' (Section 4.1): At the one end of the scale, the block-diagonal structure is located, meaning that the overall search problem consists of two disjoint subproblems with maximal intense intra-subproblem interactions while no crosssubproblem interactions exist (Fig. 1a). Hence, the level $K^{*}$ of cross-subproblem complexity is at a minimum (i.e., $K^{*}=0$ ). At the other end of the scale, there is a search problem where all single options $d_{i}$ affect the fitness contributions $C_{j}$ of all other choices; i.e., the intensity of interactions among the choices assigned to the search agents is raised to a maximum and the complexity is maximal (Fig. 1c). We call this a full interdependent interaction structure. For these and other structures see Rivkin and Siggelkow (2007).

Second, we conduct a sensitivity analysis to gain some further insights on the relevance of the search problem's complexity in the context of organizational dynamics (Section 4.2). In particular, we increase $K^{*}$ in steps of 1 and randomly select $K^{*}$ choices assigned to search agent $s(s \neq r)$ to also affect the performance achieved by agent $r$ (Fig. 1b, $\left.K^{*}=2\right)$. However, in this part of the analysis the level of subproblem complexity is kept stable for the entire observation period.

Third, we take into account that the task environment as captured in the complexity of the search problem could be unstable (Section 4.3). Hence, in this part of the analysis, the level of subproblem complexity $K^{*}$ is modified at a certain point $T^{\mathrm{c}}$ in time. In particular, we introduce results for situations in which, in the beginning, the search problem shows no subproblem interactions, and then-as a
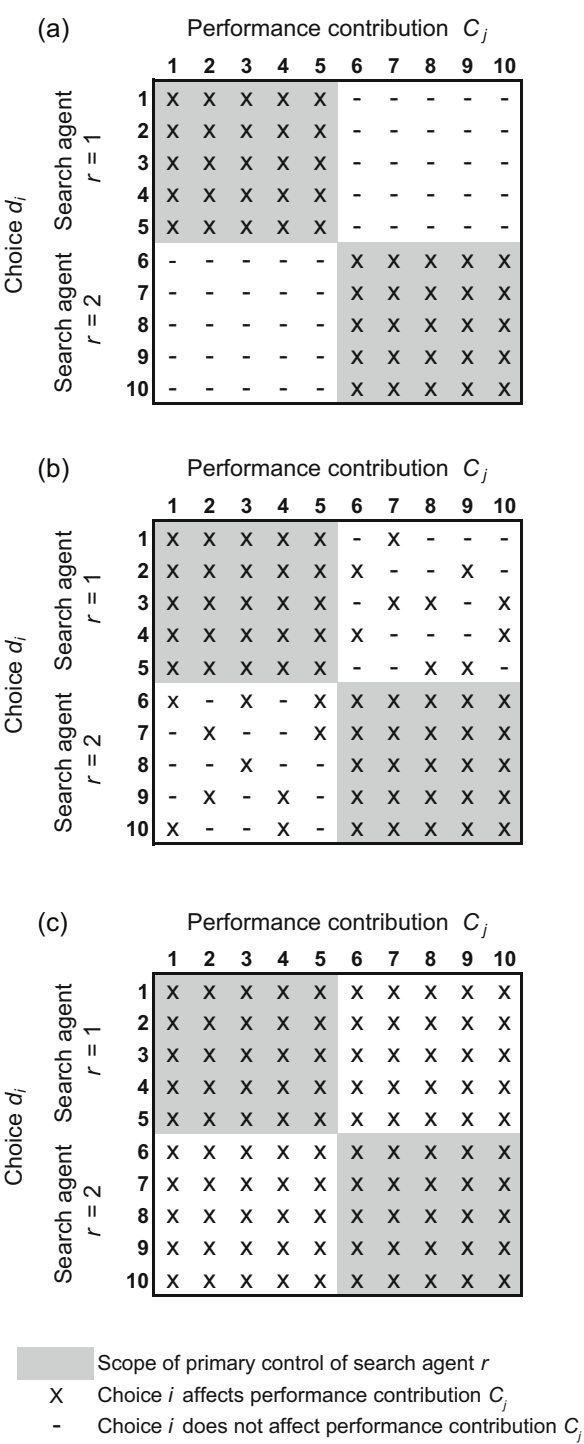

Fig. 1 Interaction structures in the simulations: (a) block-diagonal structure $\left(K=4, K^{*}=0\right)$; (b) exemplary intermediate structure $\left(K=6, K^{*}=2\right)$; (c) full interdependent structure $\left(K=9, K^{*}=5\right)$

kind of external shock - $K^{*}$ is raised at once to a higher level. The question behind these simulations is whether search systems employing organizational dynamics are more capable of dealing with unstable task environments than with search systems which remain unchanged.

The simulation model as introduced in Section 2 employs a considerable number of parameters which could lead to a rather vast space of possible combinations. To be clear and concise, we restrict our simulations to parameter settings as listed in Table 1. In the table, parameters are grouped according to the major components and steps of our simulations. 
Table 1 Parameter settings

\begin{tabular}{ll}
\hline \multicolumn{1}{c}{ Parameter } & \multicolumn{1}{c}{ Values $/$ Types } \\
\hline I. Parameters characterizing the general set-up of the search problem \\
$\quad N=10$ \\
Number of choices & $M=2 ;$ agent $1: \boldsymbol{d}^{1}=\left(d_{1}, d_{2}, \ldots, d_{5}\right) ;$ agent $2: \boldsymbol{d}^{2}=\left(d_{6}, d_{7}, \ldots, d_{10}\right)$ \\
Number of search agents & $T=200$
\end{tabular}

Parameters listed in the first group remain unchanged in all simulations presented in this paper. With respect to the observation time, it is worth mentioning that we chose $T=200$ periods due to the results of our pre-tests: pre-tests indicated that without organizational alterations and for $T>200$ the performance level achieved remains rather stable. In particular, in pre-tests we extended the observation period to $T=1000$ and found the following: A longer observation period leads to a performance enhancement of 0.0002 for the block-diagonal or 0.0004 for the full interdependent structure compared against the final performance achieved in $t=200$ which is at a level of 0.9460 or 0.8722 respectively (Table 2); at the same time the major effects of organizational dynamics become obvious even within the first 200 periods as described in Section 4.

Parameters in the second group capture the mode of changing the organizational set-up and the alternatives within each of the organizational dimensions under change. The interval of change is set to 25 periods for the 'periodical' and the 'fitness-driven' modes (for an analysis see Wall (2016b)). Obviously, the alternatives within each of the organizational dimensions reflect only some of a rather vast space of organizational design alternatives; however, the options are intended to capture rather distinct alternatives (i.e., residing rather at the end of scales as well as one intermediate option).

Parameters in the third group capture the complexity of the search problems analyzed within the three steps of our simulations as sketched in the beginning of this section.

\section{Results and discussion}

\subsection{Baseline scenarios}

Table 2 reports condensed results of the simulations of the baseline scenarios where each row represents results of 5000 adaptive walks: 1000 distinct fitness landscapes with 5 adaptive walks on each. As captured in the table, we distinguish three different measures for the effectiveness of the search since, reasonably, it may depend on the special context of the search system which of these measures is more relevant. In addition to the final performance achieved at the end of the observation period $\left(V_{t=200}\right)$, the average performance $\bar{V}_{\{0 ; 200\}}$ over the observation time is informative of the performance achieved on average in each of the $T=200$ periods. Hence, while the final performance informs whether at the end of the observation period a good performance has been achieved or not, the average performance takes the entire search process up to this point in time into account. For example, think of a firm searching for a superior configuration of its performance drivers: in this case, reasonably, it is also relevant which performance is achieved during the course of the search. $V_{t=200}$ and $\bar{V}_{\{0 ; 200\}}$ are relative measures meaning that they are given in relation to the global maximum of the respective performance landscapes (otherwise the results could not be compared across 
Table 2 Condensed results for the baseline scenarios

\begin{tabular}{|c|c|c|c|c|c|c|c|}
\hline Change mode & $\begin{array}{c}\text { Final } \\
\text { performance } \\
\left(V_{t=200}\right)\end{array}$ & $\begin{array}{l}\mathrm{CI}^{*} \text { of final } \\
\text { performance } \\
\quad\left(V_{t=200}\right)\end{array}$ & $\begin{array}{c}\text { Average } \\
\text { performance } \\
\left(\bar{V}_{\{0 ; 200\}}\right)\end{array}$ & $\begin{array}{c}\mathrm{CI}^{*} \text { of average } \\
\text { performance } \\
\left(\bar{V}_{\{0 ; 200\}}\right)\end{array}$ & $\begin{array}{c}\text { Relative } \\
\text { frequency** } \\
(t=200)\end{array}$ & $\begin{array}{c}\text { Ratio of } \\
\text { alternated } \\
\text { configurations }\end{array}$ & $\begin{array}{l}\text { Average } \\
\text { number of } \\
\text { changes }\end{array}$ \\
\hline \multicolumn{8}{|c|}{ Block-diagonal interaction structure } \\
\hline No change & 0.9460 & \pm 0.0027 & 0.9395 & \pm 0.0026 & $24.60 \%$ & $2.88 \%$ & 0 \\
\hline Once & 0.9592 & \pm 0.0022 & 0.9505 & \pm 0.0021 & $31.40 \%$ & $4.80 \%$ & 1 \\
\hline Periodical & 0.9674 & \pm 0.0020 & 0.9532 & \pm 0.0015 & $38.10 \%$ & $7.30 \%$ & 7 \\
\hline Fitness-driven & 0.9612 & \pm 0.0024 & 0.9482 & \pm 0.0017 & $34.38 \%$ & $8.07 \%$ & 4.32 \\
\hline \multicolumn{8}{|c|}{ Full interdependent interaction structure } \\
\hline No change & 0.8722 & \pm 0.0034 & 0.8630 & \pm 0.00307 & $3.96 \%$ & $4.76 \%$ & 0 \\
\hline Once & 0.8861 & \pm 0.0032 & 0.8738 & \pm 0.00290 & $5.72 \%$ & $5.49 \%$ & 1 \\
\hline Periodical & 0.8914 & \pm 0.0037 & 0.8736 & \pm 0.00243 & $7.22 \%$ & $8.54 \%$ & 7 \\
\hline Fitness-driven & 0.8769 & \pm 0.0047 & 0.8645 & \pm 0.00282 & $7.36 \%$ & $12.58 \%$ & 4.31 \\
\hline
\end{tabular}

performance landscapes). The third measure for the effectiveness of the search process reported is the relative frequency of how often the global maximum is found in the final period. Moreover, we report two measures which are mainly intended to give some information about the search process: the ratio of alternated configurations informs on the diversity of the search process, i.e., about the ratio of periods in which the search system has implemented an altered configuration $\left(\boldsymbol{d}_{t}\right)$ as compared to the immediately prior period. Additionally, the number of changes, i.e., the number of periods in which the organizational set-up was modified, is reported.

Figs. 2 and 3 display the adaptive walks for the different change modes and the interaction structures of the baseline scenarios. Table 3 reports results of comparisons of means in terms of differences between means of average performance and the significances of these differences according to Welch's method (Welch, 1938) as it it suggested for the analysis of simulations (Law, 2007).

In general, results suggest that organizational dynamics in the course of search may have beneficial effects on the performance levels achieved; however, results also indicate that the effects of alterations depend on the change mode in conjunction with the complexity of the underlying search problem.

In particular, in the block-diagonal interaction structure all three modes involving organizational dynamics clearly outperform the organizationally stable set-up with respect to all three of our measures for effectiveness of search. In the full interdependent interaction structure final and average performances

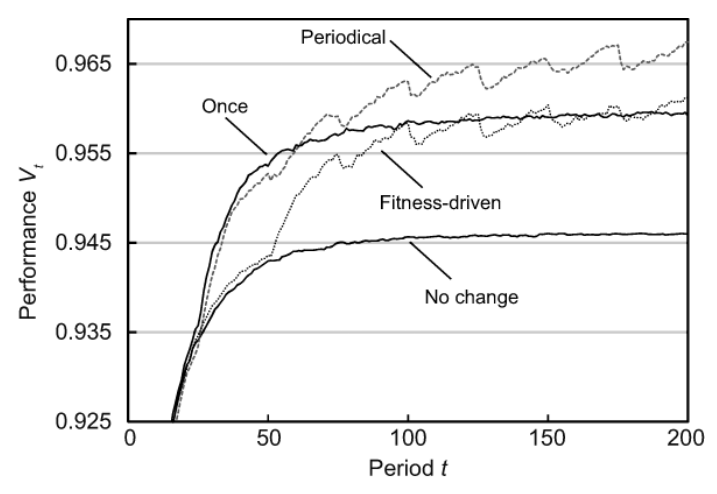

Fig. 2 Adaptive search processes in the block-diagonal interaction structure. Each curve represents the average of 5000 adaptive walks: 1000 distinct fitness landscapes with 5 adaptive walks on each. For parameter settings see Table 1. Reprinted from Wall (2015), Copyright 2015, with permission from Springer

benefit from the 'once' and the 'periodical' change modes; in contrast, in this maximally complex interaction structure the 'fitness-driven' mode provides final and average performance levels that are nearly the same as achieved without any change. However, the global maximum is found more often under the regime of the 'fitness-driven' mode than with keeping the organizational structure stable.

Hence, from these results two questions arise: (1) What causes the - generally speaking - beneficial effects of organizational change on the effectiveness of search, and (2) why do these effects apparently vary with the complexity of the interaction structure? We postpone the second question to Section 4.2.

With respect to the first question, we argue that altering the organizational set-up in the course of the 


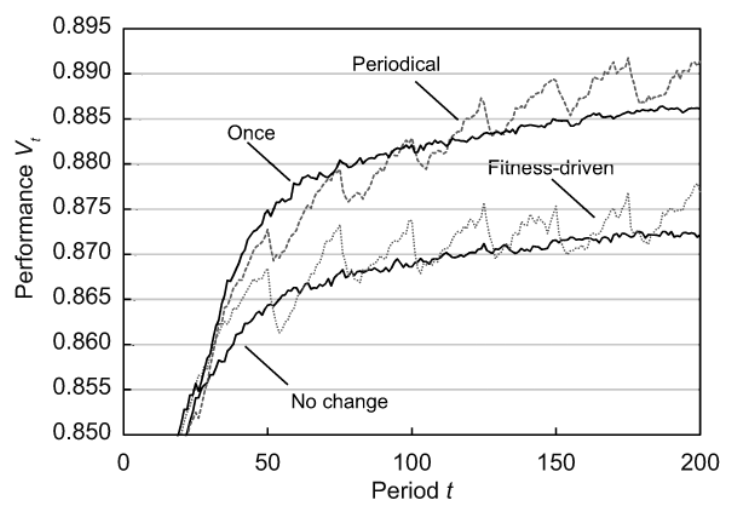

Fig. 3 Adaptive search processes in the full interdependent interaction structure. Each curve represents the average of 5000 adaptive walks: 1000 distinct fitness landscapes with 5 adaptive walks on each. For parameter settings see Table 1. Reprinted from Wall (2015), Copyright 2015, with permission from Springer

Table 3 Mean differences (up) and individual 99.9\% confidence intervals (down) for all pairwise comparisons of means of average performance $\bar{V}_{\{0 ; 200\}}$ in the baseline scenarios

\begin{tabular}{lccc}
\hline \multirow{2}{*}{ Change } & \multicolumn{2}{c}{ Mean difference \& confidence level } \\
\cline { 2 - 4 } mode & Once & Periodical & Fitness-driven \\
\hline Block-diagonal interaction & structure \\
No change & 0.0137 & 0.0110 & 0.0130 \\
& $\pm 0.0033^{*}$ & $\pm 0.0030^{*}$ & $\pm 0.0031^{*}$ \\
Once & & -0.0023 & -0.0023 \\
& & \pm 0.0026 & \pm 0.0027 \\
Periodical & & -0.0050 \\
& & & $\pm 0.0023^{*}$ \\
\hline Full interdependent interaction & structure & \\
No change & 0.0108 & 0.0106 & 0.0011 \\
& $\pm 0.0042^{*}$ & $\pm 0.0039^{*}$ & \pm 0.0040 \\
Once & -0.0002 & -0.0096 \\
& & \pm 0.0038 & $\pm 0.0039^{*}$ \\
Periodical & & & -0.0094 \\
& & & $\pm 0.0035^{*}$ \\
\hline
\end{tabular}

* indicates a significant difference

search for higher levels of performance affects the assessment of options by the search agents. In particular, after an alteration the current solution (i.e., status quo $\left.\boldsymbol{d}_{t-1}\right)$ and adjacent alternative solutions might be assessed differently than before the change. This may be due to a modified perspective on the search problem as affected by parameter $\alpha^{r}$, because the information base is modified (i.e., precision of information) or since the coordination mode induces another level of autonomy of the search agents. In consequence, after the change the preferences of the searching agents might be modified compared to the prior period and, hence, another configuration may be implemented. With this, organizational alterations provide an impulse to leave the status quo and, moreover, to potentially discover new areas in the fitness landscape (exploration). Hence, changing the organizational set-up of the search system increases the diversity of search and, thus, reduces the peril of sticking to an inferior local maximum (Siggelkow and Levinthal, 2003; Baumann, 2013). This argument is broadly supported by the ratio of periods with altered configurations $\boldsymbol{d}_{t}$ as reported in Table 2: this measure is two to three times higher than in the no-change settings.

In sum, this lets us hypothesize that inducing organizational dynamics in search processes increases the diversity of search and, by that, reduces the peril of sticking to an inferior local maximum. As becomes obvious from Figs. 2 and 3, the 'periodical' and the 'fitness-driven' modes induce oscillations with respect to the performance levels achieved over time. Reasonably, this is due to a trade-off between exploration of new areas in the fitness landscape and exploitation of the current position in the landscape. In particular, as argued above, each alteration brings along the chance to discover new options and, eventually, new areas in the fitness landscape but, by that, also may disrupt an incremental improvement in the neighborhood of the status quo.

Having said that, the question remains why the 'fitness-driven' mode shows such a rather poor performance in the highly complex search problem. Moreover, altering the organizational set-up 'once' provides a performance gain comparable to the 'periodical' mode in the full interdependent structure which is not the case in the block-diagonal structure. Since these findings also relate to the sensitivity against complexity of cross-agent interactions we postpone this discussion to the subsequent section.

\subsection{Varying the complexity of cross-agent in- teractions (sensitivity analysis)}

To gain a deeper understanding of the interference of organizational dynamics and complexity of cross-agent interactions further simulations were conducted in which the parameter $K^{*}$ was varied (Figs. 4 and 5, for parameters see Table 1).

Results indicate that effectiveness of search declines with increasing complexity as is depicted for the final performance $V_{t=200}$ in Fig. 4 which corresponds to prior research (Altenberg, 1997; Rivkin 


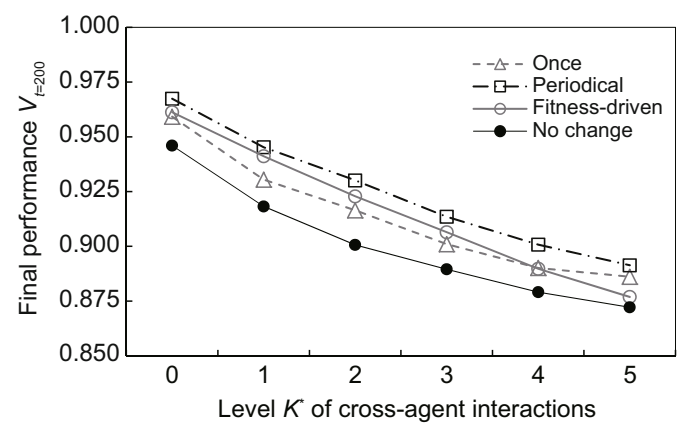

Fig. 4 Final performance achieved for different levels of complexity and different change modes. Each mark represents the result of 5000 adaptive walks: 1000 distinct fitness landscapes with 5 adaptive walks on each; confidence intervals range between 0.0020 and 0.0047 at a confidence level of 0.999 ; for parameter settings see Table 1

and Siggelkow, 2007). Decreasing shapes of curves are also obtained for average performance $\bar{V}_{\{0 ; 200\}}$ and the frequency of the global maximum found. However, with respect to our research question, it appears interesting to compare the change modes more explicitly. To this end, for each performance measure (i.e., final and average performances and frequency of global maximum found) and each level of $K^{*}$ we compute the differences between the respective results obtained for search with changes ('once', 'periodical', and 'fitness-driven') and for a search without employing change ('no change'). Results are plotted in Fig. 4 and Table 4 reports on significances of mean differences according to Welch's method (Welch, 1938).

The results indicate that, generally speaking, alterations of the organizational set-up provide significant positive contributions with respect to the three measures of effectiveness of search for the levels of complexity simulated. This becomes particularly obvious in Fig. 5 which plots the differences of employing organizational dynamics compared with 'no change' settings for various levels of $K^{*}$ and different performance measures: Performance differences are positive for all levels of $K^{*}$ and for all measures. However, some aspects are worth discussing in detail:

1. Regardless of the level of complexity, changing the organizational set-up once in the course of search apparently provides an 'add-on' of around 0.015 to the final performance and 0.01 to average performance, compared to keeping the organizational setup stable for the entire observation period. However, for low levels of complexity the 'once' mode enhances
Table 4 Mean differences (up) and individual $99.9 \%$ confidence intervals (down) for all pairwise comparisons of means of performance for different change modes against 'no change'

\begin{tabular}{|c|c|c|c|}
\hline \multirow{2}{*}{$\begin{array}{c}\text { Level of } \\
\text { complexity }\left(K^{*}\right)\end{array}$} & \multicolumn{3}{|c|}{ Mean difference \& confidence level } \\
\hline & Once & Periodical & Fitness-driven \\
\hline \multicolumn{4}{|c|}{ Final performance $\left(V_{t=200}\right)$} \\
\hline \multirow[t]{2}{*}{0} & 0.0132 & 0.0214 & 0.0152 \\
\hline & $\pm 0.0035^{*}$ & $\pm 0.0034^{*}$ & $\pm 0.0036^{*}$ \\
\hline \multirow[t]{2}{*}{1} & 0.0122 & 0.0270 & 0.0229 \\
\hline & $\pm 0.0041^{*}$ & $\pm 0.0040^{*}$ & $\pm 0.0041^{*}$ \\
\hline \multirow[t]{2}{*}{2} & 0.0158 & 0.0294 & 0.0222 \\
\hline & $\pm 0.0043^{*}$ & $\pm 0.0043^{*}$ & $\pm 0.0045^{*}$ \\
\hline \multirow[t]{2}{*}{3} & 0.0114 & 0.0240 & 0.0169 \\
\hline & $\pm 0.0045^{*}$ & $\pm 0.0046^{*}$ & $\pm 0.0050^{*}$ \\
\hline \multirow[t]{2}{*}{4} & 0.0110 & 0.0217 & 0.0107 \\
\hline & $\pm 0.0045^{*}$ & $\pm 0.0048^{*}$ & $\pm 0.0053^{*}$ \\
\hline \multirow[t]{2}{*}{5} & 0.0139 & 0.0192 & 0.0047 \\
\hline & $\pm 0.0047^{*}$ & $\pm 0.0050^{*}$ & \pm 0.0058 \\
\hline \multicolumn{4}{|c|}{ Average performance $\left(\bar{V}_{\{0 ; 200\}}\right)$} \\
\hline \multirow[t]{2}{*}{0} & 0.0110 & 0.0137 & 0.0087 \\
\hline & $\pm 0.0033^{*}$ & $\pm 0.0030^{*}$ & $\pm 0.0031^{*}$ \\
\hline \multirow[t]{2}{*}{1} & 0.0093 & 0.0152 & 0.0108 \\
\hline & $\pm 0.0039^{*}$ & $\pm 0.0035^{*}$ & $\pm 0.0036^{*}$ \\
\hline \multirow[t]{2}{*}{2} & 0.0120 & 0.0167 & 0.0112 \\
\hline & $\pm 0.0040^{*}$ & $\pm 0.0037^{*}$ & $\pm 0.0038^{*}$ \\
\hline \multirow[t]{2}{*}{3} & 0.0090 & 0.0126 & 0.0081 \\
\hline & $\pm 0.0042^{*}$ & $\pm 0.0039^{*}$ & $\pm 0.0040^{*}$ \\
\hline \multirow[t]{2}{*}{4} & 0.0076 & 0.0114 & 0.0043 \\
\hline & $\pm 0.0042^{*}$ & $\pm 0.0039^{*}$ & $\pm 0.0041^{*}$ \\
\hline \multirow[t]{2}{*}{5} & 0.0108 & 0.0106 & 0.0015 \\
\hline & $\pm 0.0042^{*}$ & $\pm 0.0039^{*}$ & \pm 0.0042 \\
\hline
\end{tabular}

* indicates a significant difference

the chance to find the global maximum remarkably.

2. Modifying the organizational set-up periodically appears to be the most beneficial change mode with respect to all measures of effectiveness. Regarding final and average performances the beneficial effect appears to be highest for a medium level of cross-agent complexity (i.e., $K^{*}=2$ ). With increasing complexity the positive effect on the frequency of global maximum found decreases from +14 to nearly +3 points of percentage.

3. The 'fitness-driven' mode appears to be rather sensitive to complexity: with increasing levels of complexity, final and average performances first benefit from increasing and then from decreasing contributions of change. For maximal complexity (i.e., $K^{*}=5$ ) there is no significant positive effect on final and average performances (Table 4).

To obtain a more detailed understanding of these results, it appears helpful to further follow the argumentation introduced in Section 4.1: 

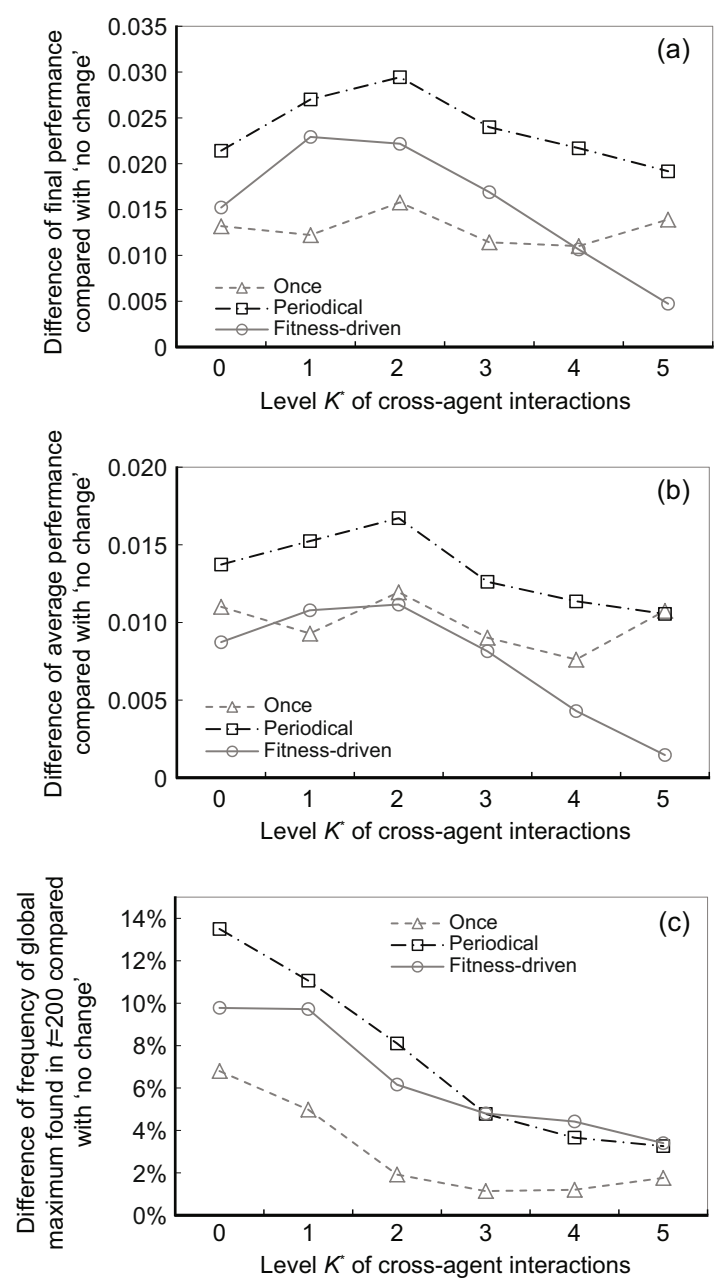

Fig. 5 Differences in effectiveness between search processes with and without change of organizational set-up. Each mark represents the difference of means where each mean result from 5000 adaptive walks: 1000 distinct fitness landscapes with 5 adaptive walks on each landscape; for parameter settings see Table 1

altering the organizational set-up in the course of the adaptive search alters the assessment of options too, and, thus, potentially directs the search to new areas in the landscape (exploration); however, this also means that 'exploitation', i.e., the process of incremental improvement in a certain area of a landscape, is interrupted - or in short: modifying the organizational set-up induces a shift from exploitation to exploration. With this, on the one hand the peril of sticking to a local maximum is reduced, which is particularly relevant for higher levels of complexity; on the other hand, for more complex structures of interactions stepwise improvement, i.e., time-consuming exploitation, is rather relevant in the search for superior levels of performance
(Siggelkow and Rivkin, 2005).

With this in mind, for repeated organizational change it appears reasonable that for increasing complexity the positive effects of more exploration first might outweigh the negative effects of interrupting exploitation until a certain critical level of complexity is reached. Beyond this critical level the negative effects (i.e., less exploitation) outweigh the positive effects (i.e., more exploration) of organizational dynamics. For the parameter settings of our simulations, this critical level of cross-agent complexity apparently is reached at $K^{*}=2$ in the "periodical' mode and at $K^{*}=1$ in the 'fitness-driven' mode (Fig. 4). Obviously, this trade-off is not relevant in the 'once' mode since here the search process for 'once' might be redirected (exploration) and afterwards no interruptions of stepwise improvements occur.

However, the question is why the 'fitness-driven' mode appears to be particularly prone to the negative effects of organizational dynamics. We argue that this is due to the fact that here the selection of situations in which alterations take place is a rather special one: the 'fitness-driven' mode, in a way, captures the impatience with settings which show low performance gains in short term. In particular, whenever the threshold $v$ of performance gain has not been achieved within a given time period $T^{* * *}$, the organizational set-up is altered. With this, on the one hand, changes are induced especially when stepwise improvement (exploitation) has not been successful so far (i.e., below threshold $v$ ) and, it may be, more time (than given by $T^{* * *}$ ) would be required to find superior levels of performance in the neighborhood. On the other hand, this mode keeps the organization as it is when stepwise improvement by chance has led to an early performance gain (and, it may be, the adaptive walk already sticks to a local maximum). To put it even more briefly: for higher levels of complexity frequent organizational change might induce too much exploration at the cost of exploitation and the 'fitness-driven' mode may select particularly those situations to induce exploration when more exploitation might be required. We find some evidence for this explanation in the ratio of those periods to the total number of observation periods in which the configuration of vector $\boldsymbol{d}_{t}$ is altered. This measure, which gives an indication for the diversity of search, is considerably higher in the 
'fitness-driven' than in the 'periodical' mode (see also Table 2).

For the design of a distributed search system and the choice of a change mode in particular, these results suggest that the complexity of cross-agent interactions $K^{*}$ should be taken into consideration: whether and, if so, to what extent, organizational dynamics is beneficial apparently depends on the level of complexity in conjunction with the temporal change mode. With this in mind, results like those presented in Fig. 4 could be regarded from a slightly different perspective: as indicated in the right most column of Table 2, the different change modes employ different numbers of changes (e.g., for our parameter settings in the 'periodical' mode the set-up is altered seven times). It depends on the particular context - to what extent changing the organizational set-up also induces 'costs': for example, in a swarm of UAVs altering the organizational set-up might just mean switching between routines/algorithms already implemented - causing nearly no marginal costs; but think, for example, of a firm undergoing an organizational change process which, certainly, does not come without cost. Then the costs of change should be considered too and, in particular, related to the benefits of change. Hence, if the designer of the search system is informed about the level of crossagent complexity, results in principle similar to those plotted in Fig. 4 could inform the designer about the benefit of the respective change mode which is not to be exceeded by the costs of change.

\subsection{Instability of complexity of cross-agent interactions}

An interesting question is whether search systems employing organizational dynamics are more capable in dealing with unstable task environments than systems which keep their organizational set-up unchanged. In our modeling effort the task environment is captured by the level of complexity $K$ of the search problem, and - given our research questionby the complexity of cross-agent interactions $K^{*}$ in particular. Hence, we conducted some exemplary simulations in which at $t=T^{c}=50$ the level of cross-agent interactions was raised from $K^{*}=0$ (i.e., the block-diagonal structure) to level $0<K^{*} \leq 5$. The increase in complexity may be caused by an external shock (for example, an innovation leading to new technological interdependencies between modules delegated to different designers, i.e., search agents).

Fig. 6 displays results for the different measures of effectiveness. For the observation time given at $T=200$, results indicate that periodical changes provide a relatively robust gain in final and average performances compared to the 'no change' mode. Note that, here we refrain from introducing results for the 'once' mode since the results obviously are subject to the timing of the singular organizational change event and - given the parameter settings in Table 1 - with $T^{*}=25$ and $T^{\mathrm{c}}=50$ merely a difference between 'no change' and 'once' in average performance occurs, which results from the 'preshock' part of the adaptive walk. With respect to the
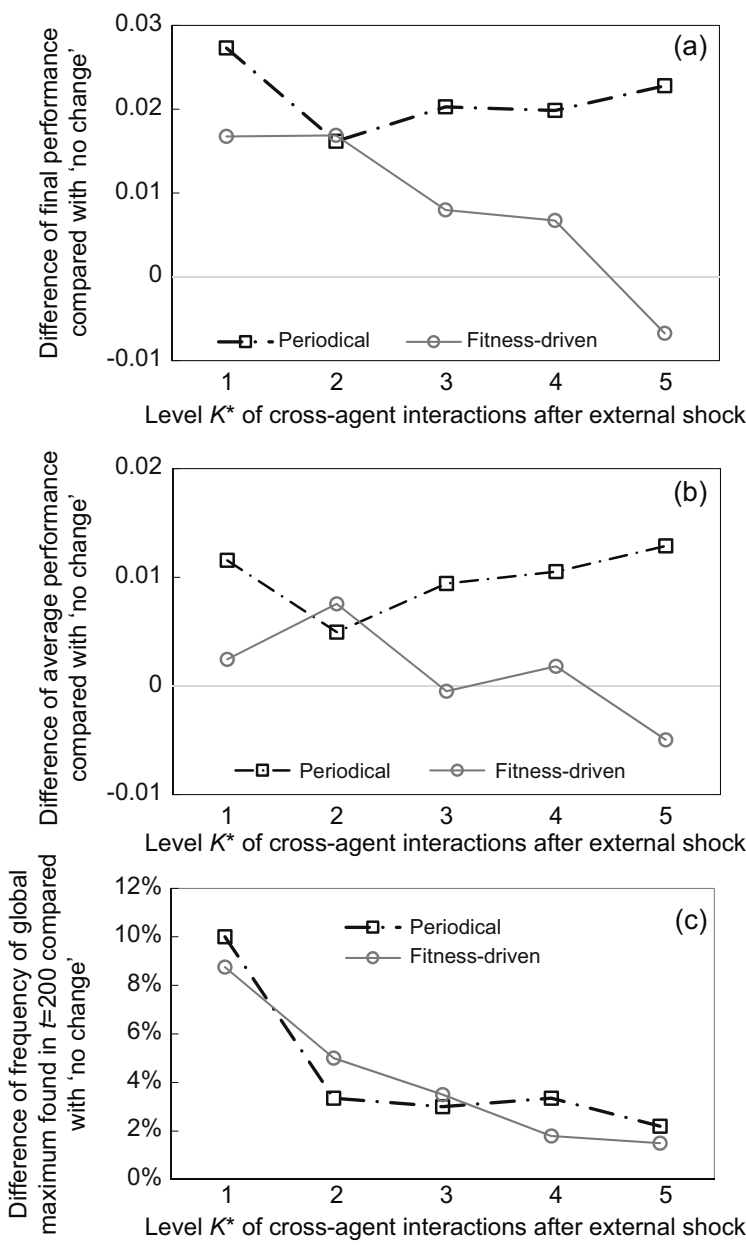

Fig. 6 Differences in effectiveness between search processes with and without change of organizational set-up. Each mark represents differences of means where each mean result from 2000 adaptive walks on two distinct fitness landscapes in each walk: a pre-shock and a post-shock landscape; for parameter settings see Table 1 
frequency of the global maximum found, periodical changes yield a rather stable advantage for all levels $1<K^{*} \leq 1$. In contrast, employing a 'fitness-driven' mode of change does not universally provide a gain of effectiveness compared to keeping the organizational set-up unchanged for the entire observation period, particularly for high levels of $K^{*}$, which corresponds to the results obtained for the full interdependent interaction structure as presented in Section 4.1.

Hence, in sum these results provide some evidence for the beneficial effects of inducing organizational dynamics in distributed systems, in particular, for unstable task environments; however, these effects appear to depend subtly on the level of (change in) complexity and the mode of organizational change employed.

\section{Conclusions}

The major findings of our study are that modifying the organizational set-up of distributed adaptive search processes has the potential to increase the effectiveness of search, and that however, the temporal mode of change in conjunction with the complexity of the search problem considerably affects the effectiveness of organizational dynamics. In particular, organizational dynamics reasonably induces a shift towards less exploitation and more exploration in the search processes, which is differently beneficial for different levels of complexity of the search problem.

With this, the findings could also provide some guidance for the design of distributed search systems. In particular, it becomes obvious that knowledge about the interactions between subproblems assigned to search agents is helpful for deciding on an adequate temporal change mode: The level of complexity apparently shapes the beneficial effects of the change modes which, for their part, shape the number of alterations of the organizational set-up and, by that, the cost effects of organizational dynamics.

Of course, the analysis presented in this paper is subject to several limitations which should be overcome in further research. For example, the change modes analyzed so far are rather simple ones and an obvious extension of our research is to study the effects of learning about (more or less successful) organizational changes in the course of search processes. Another interesting extension would be, for example, to adapt our model to certain contexts which would allow going into more detail on the cost effects of organizational changes and, hence, the net benefit of organizational dynamics.

\section{References}

Altenberg, L., 1997. NK fitness landscapes. In: Back, T., Fogel, D.B., Michalewicz, Z. (Eds.), Handbook of Evolutionary Computation. Oxford University Press, UK, p.B2.7:5-B2.7:10.

Baumann, O., 2013. Distributed problem solving in modular systems: the benefit of temporary coordination neglect. Syst. Res. Behav. Sci., 32(1):124-136. http://dx.doi.org/10.1002/sres.2218

Cao, Y., Yu, W., Ren, W., et al., 2013. An overview of recent progress in the study of distributed multi-agent coordination. IEEE Trans. Ind. Inform., 9(1):427-438. http://dx.doi.org/10.1109/TII.2012.2219061

Carley, K.M., Gasser, L., 1999. Computational organization theory. In: Weiss, G. (Ed.), Multiagent Systems: a Modern Approach to Distributed Artificial Intelligence. MIT Press, Cambridge, p.299-330.

Gross, T., Blasius, B., 2008. Adaptive coevolutionary networks: a review. J. Royal Soc. Interf., 5(20):259-271. http://dx.doi.org/10.1098/rsif.2007.1229

Hansen, M.T., 1999. The search-transfer problem: the role of weak ties in sharing knowledge across organization subunits. Administr. Sci. Quart., 44(1):82-111. http://dx.doi.org/10.2307/2667032

Karp, R.M., Upfal, E., Wigderson, A., 1988. The complexity of parallel search. J. Comput. Syst. Sci., 36(2):225253. http://dx.doi.org/10.1016/0022-0000(88)90027-X

Kauffman, S., 1993. The Origins of Order: Self-Organization and Selection in Evolution. Oxford University Press, UK.

Kauffman, S., Levin, S., 1987. Towards a general theory of adaptive walks on rugged landscapes. J. Theor. Biol., 128(1):11-45.

http://dx.doi.org/10.1016/S0022-5193(87)80029-2

Law, A., 2007. Simulation Modeling and Analysis. McGrawHill, USA.

Levitan, B., Kauffman, S., 1995. Adaptive walks with noisy fitness measurements. Mol. Divers., 1(1):53-68. http://dx.doi.org/10.1007/BF01715809

Li, R., Emmerich, M.T.M., Eggermont, J., et al., 2006. Mixed-integer NK landscapes. Proc. 9th Int. Conf. on Parallel Problem Solving from Nature, p.42-51. http://dx.doi.org/10.1007/11844297_5

Peterson, C., 1990. Parallel distributed approaches to combinatorial optimization: benchmark studies on traveling salesman problem. Neur. Comput., 2(3):261-269. http://dx.doi.org/10.1162/neco.1990.2.3.261

Rivkin, J.W., Siggelkow, N., 2007. Patterned interactions in complex systems: implications for exploration. Manag. Sci., 53(7):1068-1085. http://dx.doi.org/10.1287/mnsc. 1060.0626

Siggelkow, N., Levinthal, D.A., 2003. Temporarily divide to conquer: centralized, decentralized, and reintegrated organizational approaches to exploration and adaptation. Organ. Sci., 14(6):650-669. 
Siggelkow, N., Rivkin, J.W., 2005. Speed and search: designing organizations for turbulence and complexity. Organ. Sci., 16(2):101-122.

http://dx.doi.org/10.1287/orsc.1050.0116

Thompson, J.D., 1967. Organizations in Action: Social Science Bases of Administrative Theory. McGraw-Hill, USA.

Wall, F., 2010. The (beneficial) role of informational imperfections in enhancing organisational performance. Progress in Artificial Economics, p.115-126. http://dx.doi.org/10.1007/978-3-642-13947-5 10

Wall, F., 2013. Comparing basic design options for management accounting systems with an agent-based simulation. Proc. 10th Int. Conf. on Distributed Computing and Artificial Intelligence, p.409-418. http://dx.doi.org/10.1007/978-3-319-00551-5 50

Wall, F., 2015. Effects of organizational dynamics in adaptive distributed search processes. Proc. 12th Int. Conf. on Distributed Computing and Artificial Intelligence, p.121-128.

http://dx.doi.org/10.1007/978-3-319-19638-1_14

Wall, F., 2016a. Agent-based modeling in managerial science: an illustrative survey and study. Rev. Manag. Sci., 10(1):135-193.

http://dx.doi.org/10.1007/s11846-014-0139-3

Wall, F., 2016b. Beneficial effects of randomized organizational change on performance. Adv. Complex Syst., 18(5-6):1550019.1-1550019.23. http://dx.doi.org/10.1142/S0219525915500198

Welch, B.L., 1938. The significance of the difference between two means when the population variances are unequal. Biometrika, 29(3/4):350-362. 